\title{
Chromium phosphide CrP as highly active and stable electrocatalysts for oxygen electroreduction in alkaline media
}

Junfeng Liu, ${ }^{a}$ Xiaoting Yu, ${ }^{a}$ Ruifeng Du, ${ }^{a}$ Chaoqi Zhang, ${ }^{a}$ Ting Zhang, ${ }^{b}$ Jordi Llorca, ${ }^{c}$ Jordi Arbiol, ${ }^{b, d}$ Ying Wang, ${ }^{e, *}$ Michaela Meyns, ${ }^{\mathrm{a}, \#, *}$ Andreu Cabot ${ }^{\mathrm{a}, \mathrm{d}, *}$

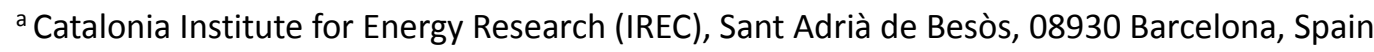

${ }^{b}$ Catalan Institute of Nanoscience and Nanotechnology (ICN2), CSIC and BIST, Campus UAB, Bellaterra, 08193 Barcelona, Catalonia, Spain

c Institute of Energy Technologies, Department of Chemical Engineering and Barcelona Research Center in Multiscale Science and Engineering, Universitat Politecnica de Catalunya, Eduard Maristany 10-14, 08019 Barcelona, Spain

d ICREA, Pg. Lluis Companys 23, 08010 Barcelona, Catalonia, Spain

e State Key Laboratory of Rare Earth Resource Utilization, Changchun Institute of Applied Chemistry, Chinese Academy of Sciences, Changchun 130022, P. R. China

\# Current address: Alfred-Wegener-Institute, Helmholtz Centre for Polar and Marine Research, Biologische Anstalt Helgoland, Kurpromenade 201, 27498 Helgoland, Germany

* Corresponding Authors

Y. Wang: ywang_2012@ciac.ac.cn,

M. Meyns: michaela.meyns@awi.de,

A. Cabot: acabot@irec.cat

\section{ABSTRACT}

Catalysts for oxygen reduction reaction (ORR) are key components in emerging energy technologies such as fuel cells and metal-air batteries. Developing low-cost, high performance and stable electrocatalysts is critical for the extensive implementation of these technologies. Herein, we present the first procedure to prepare colloidal chromium phosphide CrP nanocrystals. We further test for the first time the performance of this material, combined with carbon, as ORR electrocatalyst. CrP-based catalysts exhibited remarkable activities with a limiting current density of $4.94 \mathrm{~mA} \mathrm{~cm}^{-2}$ at $0.2 \mathrm{~V}$, a half-potential of $0.65 \mathrm{~V}$ and an onset potential of $0.8 \mathrm{~V}$ at $1600 \mathrm{rpm}$, which are comparable to commercial Pt/C. Advantageously, CrP-based catalysts displayed much higher stabilities and higher tolerances to methanol in alkaline solution. Using DFT calculation, we demonstrate CrP to provide a very strong chemisorption of $\mathrm{O}_{2}$ which facilitates its reduction and explains the excellent ORR performance experimentally obtained for this material. 


\section{INTRODUCTION}

Proton exchange membrane fuel cells and metal air batteries are actively investigated as promising clean energy conversion and storage technologies. Common to both emerging technologies is the use of an oxygen cathode where ambient oxygen is reduced to $\mathrm{OH}^{-}$groups in solution. This reaction requires the participation of 4 electrons, what makes it very sluggish and translates in a limitation of the overall performance of the system. To accelerate this reaction, Pt or Pt-based alloys are generally required [1-4], what strongly increases the device cost and constitutes one of the major barriers toward commercialization. Hence, considerable efforts are involved in the design and production of ORR catalysts not based on Pt or Pt-group metals. Optimum ORR catalysts have been found within most material families, including metal oxides [5,6], carbides [7-9], phosphides [10-17], chalcogenides [18] and even metal-free catalysts [19-21] etc. Among them, phosphides are particularly interesting due to their stability, excellent electrical conductivities and abundance and low cost of phosphorous. Within this family, $\mathrm{PdP}_{2}$ [14], $\mathrm{Co}_{2} \mathrm{P}$ [15], $\mathrm{CoP}$ [16] and $\mathrm{Ru}_{2} \mathrm{P}$ [17] have already demonstrated excellent ORR activities, comparable to commercial Pt catalysts.

However, among the metal phosphides, chromium phosphide has been surprising ignored in most of its potential technological applications in spite of its a priori suitable properties. This is particularly shocking taking into account the relatively high abundance of chromium in the earth crust, above that of nickel, zinc or copper for example, and its high global annual production due to their use in metallurgy, what makes it a relatively low cost material. Chromium phosphide is a hard-wearing metallic conductor displaying a high thermal stability, good resistivity to oxidation and anti-corrosion toward water and dilute acids [22-28]. These properties make CrP an appealing candidate in a wide range of applications. However, very few papers have reported the synthesis and applications of chromium phosphides [22-24]. In particular, CrP has been produced in film form by chemical vapour deposition $[22,23]$, but never in the form of nanoparticles.

In a previous work, we showed that triphenyl phosphite (TPP) can be used as a low-cost and air-stable phosphorous precursor to produce a range of binary and ternary metal phosphides such as $\mathrm{Ni}_{2} \mathrm{P}, \mathrm{Co}_{2} \mathrm{P}, \mathrm{Fe}_{2} \mathrm{P}, \mathrm{Cu}_{3} \mathrm{P}, \mathrm{MoP}$ and $\mathrm{Ni}_{2}$ ${ }_{x} \mathrm{CO}_{x} \mathrm{P}[29,30]$. However, due to the low-boiling point of the presented procedure attempts to produce metal phosphides that required higher crystallization temperatures were not successful.

In the present work, we demonstrate the production of CrP nanocrystals (NCs) using TPP as a highly suitable phosphorous precursor employing a high boiling point solvent. The produced CrP NCs were combined with carbon 
and tested as electrocatalyst for ORR. Through an exhaustive investigation, we demonstrate CrP-based catalysts to be highly suitable for oxygen electroreduction.

\section{EXPERIMENTAL SECTION}

Chemicals: Triphenyl phosphite (TPP, 99\%) was purchased from Alfa Aesar. Chromium hexacarbonyl $\left(\mathrm{Cr}(\mathrm{CO})_{6}, 99+\%\right)$ and oleylamine (OAm, approximate C18 content 80-90\%) were purchased from ACROS Organics. Carbonsupported Pt NCs (Pt/C, 20 wt\% Pt), Nafion (5 wt\% in a mixture of low aliphatic alcohols and water) and potassium hydroxide $(\mathrm{KOH}, 85 \%)$ were purchased from Sigma Aldrich. Chloroform, acetone and ethanol were of analytical grade and obtained from various sources. Milli-Q water was supplied by a PURELAB flex from ELGA. All chemicals were used as received without further purification.

Synthesis of CrP NCs: All reactions were carried out under argon atmosphere using standard Schlenk line techniques. In a typical synthesis, $10 \mathrm{~mL}$ of OAm and $1 \mathrm{~mL}$ of TPP were mixed in a $50 \mathrm{~mL}$ three-neck flask equipped with a condenser and a stir bar. The system was heated to $120^{\circ} \mathrm{C}$ under Ar flow and maintained at this temperature for at least $1 \mathrm{~h}$. Then, $110 \mathrm{mg}(0.5 \mathrm{mmol})$ of $\mathrm{Cr}(\mathrm{CO})_{6}$ was quickly added into the flask under Ar flow. The temperature was then increased to $320^{\circ} \mathrm{C}$ in 20 min and kept at that temperature for $2 \mathrm{~h}$. Afterward, the mixture was allowed to cool down to room temperature by removing the heating mantle. Excess ethanol was added to the black product followed by centrifugation at $5000 \mathrm{rpm}(3200 \mathrm{~g})$ for $5 \mathrm{~min}$. Purification was achieved by another twice dispersion/precipitation steps using 1:3 (v:v) chloroform/ethanol. Finally the supernatant was discarded and the precipitated material was collected and dried in ambient conditions.

Material characterization: Transmission electron microscopy (TEM) characterization was carried out using a ZEISS LIBRA 120, operating at $120 \mathrm{kV}$ and a JEOL 1011 operating at $100 \mathrm{kV}$. Carbon-coated TEM grids from Ted-Pella were used as substrates. Powder X-ray diffraction (XRD) patterns were collected directly from the as-synthesized NPs dropped on a Si (501) substrate using a Bruker-AXS D8 Advanced X-ray diffractometer with Ni-filtered (2 $\mu \mathrm{m}$ thickness) Cu K radiation ( $\lambda=1.5406 \AA$ ) operating at $40 \mathrm{kV}$ and $40 \mathrm{~mA}$. A LynxEye linear position-sensitive detector was used in reflection geometry. High-resolution TEM (HRTEM) studies were conducted using a field emission gun FEI Tecnai F20 microscope at $200 \mathrm{kV}$ with a point-to-point resolution of $0.19 \mathrm{~nm}$. High angle annular dark-field (HAADF) STEM was combined with electron energy loss spectroscopy (EELS) in the Tecnai F20, by using a GATAN QUANTUM filter. Scanning electron microscopy (SEM) analyses were carried out using a ZEISS Auriga microscope with an energy dispersive X-ray spectroscopy (EDS) detector operating at $20 \mathrm{kV}$. Dispersive spectrometer JobinYvon Lab Ram HR 800 with Olympus BXFM microscope optic was used to obtain Raman spectra. X-ray 
photoelectron spectroscopy (XPS) was carried out on a SPECS system equipped with an Al anode XR50 source operating at $150 \mathrm{~mW}$ and a Phoibos 150 MCD-9 detector. The pressure in the analysis chamber was below $10^{-7}$ Pa. The area analyzed was about $2 \mathrm{~mm} \times 2 \mathrm{~mm}$. The pass energy of the hemispherical analyzer was set at $25 \mathrm{eV}$ and the energy step was set at $0.1 \mathrm{eV}$. Data processing was performed with the CasaXPS program (Casa Software Ltd., UK). Binding energy values were corrected using the $\mathrm{C} 1 \mathrm{~s}$ peak at $284.8 \mathrm{eV}$. Fourier transform infrared spectroscopy (FTIR) was performed on an Alpha Bruker FTIR spectrometer with a platinum attenuated total reflectance (ATR) single reflection module.

Electrochemical measurements: The as-synthesized CrP NPs were mixed with carbon black (Vulcan XC-72) with a weight ratio of $30 \%$ in a mixture chloroform and ethanol (1:1). The $\mathrm{CrP}$ and carbon mixture was sonicated for $1 \mathrm{~h}$ and then washed several times with chloroform and acetone. The precipitate was dried in air and then annealed at $450{ }^{\circ} \mathrm{C}$ under $5 \% \mathrm{H}_{2} / \mathrm{Ar}$ for $2 \mathrm{~h}$ to remove surface ligand. The catalysts ink were prepared by mixing $5 \mathrm{mg}$ of the annealed $\mathrm{CrP} / \mathrm{C}$ composite, $1 \mathrm{~mL}$ of 1:1 (v:v) deionized water/isopropanol and $17.5 \mu \mathrm{L}$ of 5 wt\% of Nafion to form a homogenous ink by sonicating for $1 \mathrm{~h}$. Subsequently, $5 \mu \mathrm{L}$ of the suspension was deposited onto a cleaned glassy carbon (GC, $3 \mathrm{~mm}$ in diameter) rotating disk electrode (RDE) with a geometric area of $0.07 \mathrm{~cm}^{2}$, and dried in ambient conditions before electrochemical measurements. For comparison, electrodes based on commercial Pt/C (20 wt\% Pt) catalyst were also fabricated following the above process.

Electrochemical measurements were conducted at room temperature on a BioLogic Electrochemical workstation using a standard three-electrode cell with the fabricated GC electrode as working electrode, Pt mesh as counter electrode and $\mathrm{Hg} / \mathrm{HgO}$ as reference electrode. The linear sweep voltammetry (LSV) curves for ORR measurements were conducted from 0.2 to $-0.7 \mathrm{~V}$ versus $\mathrm{Hg} / \mathrm{HgO}$ at a scan rate of $5 \mathrm{mV} \mathrm{s}^{-1}$ with different rotating rates in $\mathrm{O}_{2}-$ saturated $0.1 \mathrm{M} \mathrm{KOH}$ electrolyte. The cyclic voltammetry (CV) measurements were performed at a scan rate of 5 $\mathrm{mV} \mathrm{s}^{-1}$ without rotating in $\mathrm{O}_{2}$ or Ar-saturated $0.1 \mathrm{M} \mathrm{KOH}$ electrolyte. To evaluate the catalyst durability, chronoamperometry was collected at $-0.3 \mathrm{~V}$ versus $\mathrm{Hg} / \mathrm{HgO}$ in $\mathrm{O}_{2}$-saturated electrolyte with a rotating rates of 200 rpm. Methanol tolerance measurements were conducted under the same condition as chronoamperometric tests but added $3 \mathrm{M}$ methanol at 1000 s and for comparison, another same amount of $\mathrm{H}_{2} \mathrm{O}$ was added at 2000 s. As a reference electrode, $\mathrm{Hg} / \mathrm{HgO}$ calibrated with respect to the reversible hydrogen electrode (RHE) in all measurements (+ $0.866 \mathrm{~V}$ vs RHE) was used.

Density functional theory (DFT): The electronic structure and energy calculations were carried out by the spinpolarized DFT using the Vienna ab initio simulation package (VASP) [31-34]. The projector augmented wave (PAW) 
potentials were used to describe ion cores and valence electrons interactions $[35,36]$. The adopted exchangecorrelation functional was the generalized gradient approximation (GGA) with the Perdew-Burke-Ernzerhof [37]. A kinetic energy cut off of $350 \mathrm{eV}$ was used with a plane-wave basis set. The integration of the Brillouin zone was conducted using a $1 \times 1 \times 1$ Monkhorst-Pack grid [38]. The last two layers were fixed and all the other atoms were fully relaxed until the force was converged to $0.05 \mathrm{eV} / \AA ̊$ and the total energy was converged to $1.0 \times 10^{-4} \mathrm{eV} /$ atom.

A (211) surface was cut based on the bulk structure of $\mathrm{CrP}$, and a model with $1 \times 2$ unit cell and 6 layers was established. To avoid the image interactions sufficiently large vacuum of $10.0 \AA$ has been taken along the z-axis.

The adsorption energy $\left(E_{\text {ads }}\right)$ of nitrobenzene was defined as follows:

$$
E_{\text {ads }}=E_{\text {substrate+adsorbates }}-E_{\text {adsorbates }}-E_{\text {substrate }}
$$

where $E_{\text {substrate+adsorbates, }} E_{\text {adsorbates, }}$ and $E_{\text {substrate }}$ are the total energies of adsorbate molecules and the CrP substrate, a gas phase adsorbate, and an isolated substrate, respectively. A negative value indicates an exothermic chemisorption.

The free energy diagrams of the oxygen reduction reactions were evaluated by the method of Nørskov et al. [39]. Free energy change from initial states to final states of the reaction was calculated according to the following equation:

$$
\Delta G=\Delta E+\Delta Z P E-T \Delta S+\Delta G_{U}+\Delta G_{p H}+\Delta G_{f i e l d}
$$

where $\Delta \mathrm{E}$ was the energy difference between reactants and products, obtained from DFT calculations; $\Delta \mathrm{ZPE}$ and $\Delta \mathrm{S}$ were the energy differences in zero-point energy and entropy; T was the temperature and $298.15 \mathrm{~K}$ was considered; $\Delta G_{U}=e U$, where $U$ was the electrode potential with respect to standard hydrogen electrode and $e$ was transferred charge; $\Delta G_{p H}$ was defined as $k_{B} T \ln 10 \times p H$, where $k_{\mathrm{B}}$ was the Boltzmann constant. In this study $p H=13$ was chosen for alkaline medium according to experimental condition $\left(0.1 \mathrm{M} \mathrm{KOH}\right.$ solution). $\Delta G_{\text {field }}$ was the free energy correction resulted from the electrochemical double layer, which was neglected in the present study according to the previous studies [39]. The free energy of $\mathrm{H}_{2} \mathrm{O}$ was calculated in the gas phase with a pressure of $0.035 \mathrm{bar}$, which was the equilibrium vapor pressure of $\mathrm{H}_{2} \mathrm{O}$ at $298.15 \mathrm{~K}$. The free energy of $\mathrm{O}_{2}$ was obtained from the free energy change of the reaction $\mathrm{O}_{2}+2 \mathrm{H}_{2} \rightarrow 2 \mathrm{H}_{2} \mathrm{O}$, which was $-4.92 \mathrm{eV}$ at $298.15 \mathrm{~K}$ and a pressure of 0.035 bar. According to a computational hydrogen electrode model suggested by Nørskov et al. [39] the free energy of $\left(\mathrm{H}^{+}+\mathrm{e}^{-}\right)$in solution at standard conditions was assumed as the energy of $1 / 2 \mathrm{H}_{2}$. The free energy of $\mathrm{OH}^{-}$was derived 
from the reaction of $\mathrm{H}^{+}+\mathrm{OH}^{-} \rightarrow \mathrm{H}_{2} \mathrm{O}$, which was in equilibrium in water solution [40]. The entropies and vibrational frequencies of $\mathrm{O}_{2}, \mathrm{H}_{2}$, and $\mathrm{H}_{2} \mathrm{O}$ in gas phase were taken from the NIST database [41]. Zero-point energy and entropies of the adsorbed species were estimated from the vibrational frequencies. In these frequencies calculations, the substrate of $\mathrm{HL}$ or $\mathrm{HN}$ sheet was fixed.

\section{RESULTS AND DISCUSSION}

CrP NPs were produced from the reaction of chromium hexacarbonyl and triphenyl phosphite in oleylamine, as detailed in the experimental section. Figure 1a shows a representative TEM micrograph of the NPs produced following this procedure. NPs showed spheroidal geometry with an average size of $17 \pm 3 \mathrm{~nm}$ (Figure 1b). XRD patterns showed the NPs to have the CrP orthorhombic phase, with pnma space group (Figure 1f). HRTEM analysis confirmed their orthorhombic phase with $a=5.3600 \AA, b=3.1140 \AA$ and $c=6.0180 \AA$ (Figure 1c-e). Annular dark field scanning TEM (ADF-STEM) and STEM-EELS elemental composition maps revealed all NPs contained Cr and P and to have both elements uniformly distributed throughout each NP (Figure 1g). No secondary phases were detected by XRD, HRTEM and STEM-EELS analyses. Extensive SEM-EDX analysis showed the as-synthesized CrP NPs to have an excess of phosphorous, $\mathrm{P} / \mathrm{Cr}=1.4$ (Figure S1), which could be attributed to the presence of phosphorous precursor as ligand on the NPs surface. 

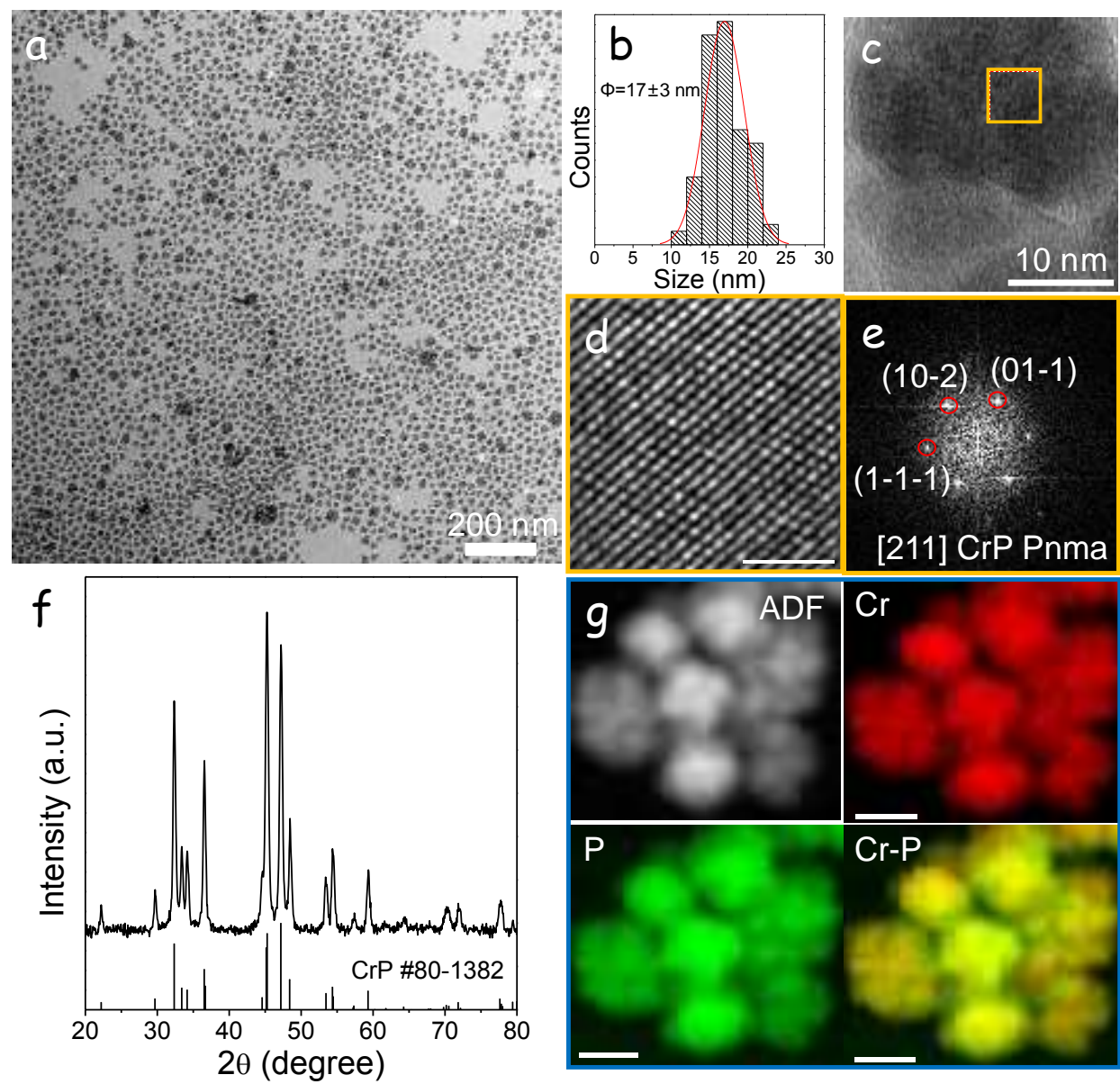

Figure 1. (a) Representative TEM micrograph of CrP NPs. (b) Histogram for the measured particle size distribution $(17 \pm 3 \mathrm{~nm})$. (c) HRTEM image of a single CrP NP. (d) Detail of the squared regions of the single CrP NPs. (e) Its respective power spectrum fitting with the CrP orthorhombic phase. (f) XRD pattern of CrP NPs including the JCPDS 80-1382 reference. (g) Annular dark field scanning TEM (ADF-STEM) image of some CrP NPs and areal density of each of the elements extracted from the EELS spectrum image.

Figure 2 shows the $\mathrm{Cr} 2 \mathrm{p}, \mathrm{P} 2 \mathrm{p}$ and valence band region of the XPS spectra of CrP NPs that had been exposed to air before measurement. The $\mathrm{Cr} 2 \mathrm{p}$ region showed $\mathrm{Cr}$ to be present at the NP surface in three different chemical states. Approximately $30 \%$ of the detected $\mathrm{Cr}$ was found in a chemical state compatible with $\mathrm{CrP}$ ( 575 and $583.9 \mathrm{eV}$ ). The other two chemical environments, $\mathrm{a} \mathrm{Cr}^{3+}(578.0$ and $587.6 \mathrm{eV})$ and $\mathrm{a} \mathrm{Cr}^{6+}(579.8$ and $589.4 \mathrm{eV})$, were associated with a slight surface oxidation of the NPs to a $\mathrm{Cr}(\mathrm{III})$ phosphate, $\mathrm{Cr}_{2} \mathrm{O}_{3}$ and $\mathrm{CrO}_{3}$ or $\mathrm{CrO}_{2}(\mathrm{OH})_{2}$ species [42-44]. Two P chemical states were identified from XPS analysis of the P $2 p$ electron states. A P 2 $p_{3 / 2}$ peak at 130.0 eV matched well with $\mathrm{P}$ in a metal phosphide environment such as CrP. This component accounted for $39 \%$ of the $\mathrm{P}$ in the outermost 2-3 nm surface of the NPs. The second component, accounting for $61 \%$ of the P detected, was found at 
a higher binding energy, $134.1 \mathrm{eV}$ for $2 \mathrm{p}_{3 / 2}$, which we assigned to a phosphate environment emerging from the partial oxidation of the surface of the CrP NPs when exposed to air. The atomic ratio of P and Cr at the NP surface detected by XPS was $\mathrm{P} / \mathrm{Cr}=2.65$, suggesting a highly P-rich NP surface, which was consistent with the confirmed excess $\mathrm{P}$ on the NPs by EDX measurement. The excess $\mathrm{P}$ on the NP surface may be derived from the phosphorous ligand on the NPs surface. As shown in figure 2c, a significant density of occupied states can be detected by XPS at the material Fermi level, which probes the metallic or highly degenerated character of the produced CrP.
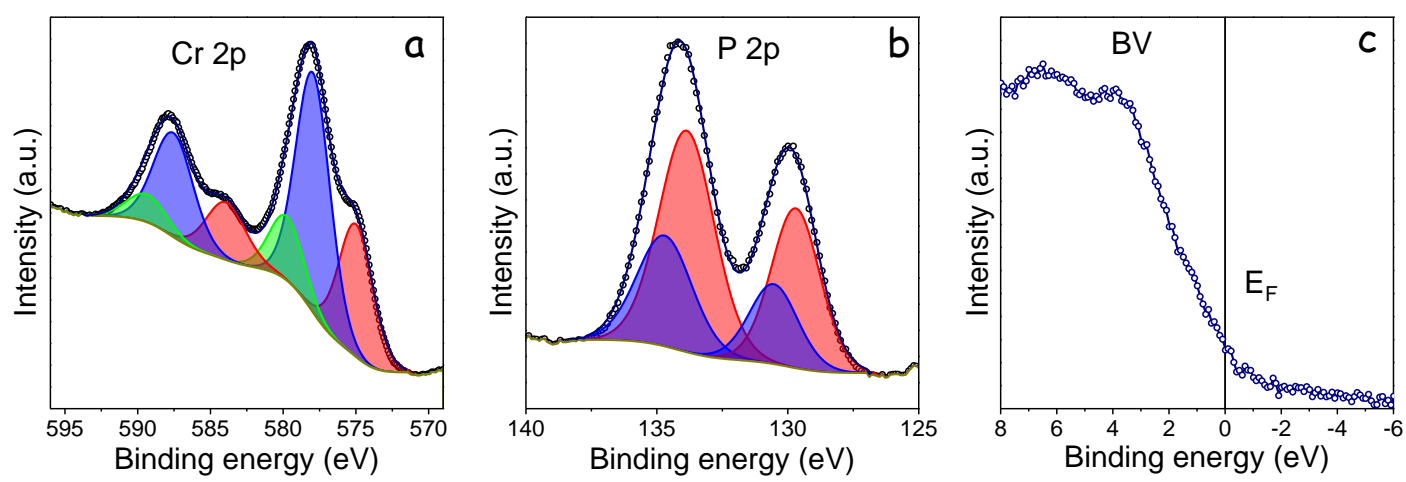

Figure 2. $\operatorname{Cr} 2 p(a), P 2 p(b)$ and valence band (c) regions of the XPS spectrum of CrP NPs.

The presence of OAm and phosphorous ligand on the NP surface was confirmed by FTIR analysis. As shown in Figure 3, the FTIR spectrum of the as-produced CrP NPs displayed peaks at 2906 and $2839 \mathrm{~cm}^{-1}$ attributed to the $\mathrm{C}-\mathrm{H}$ stretching vibration of the alkyl group of OAm. Additionally, peaks attributed to the bending vibration of $\mathrm{N}-\mathrm{H}$ at $1594 \mathrm{~cm}^{-1}$ and $\mathrm{C}-\mathrm{H}$ at $1439 \mathrm{~cm}^{-1}$ were also identified. These features were slightly shifted compared with pure OAm, consistently with its binding to the NPs surface. A peak at $3130 \mathrm{~cm}^{-1}$ was assigned to the stretching vibration of $=\mathrm{CH}$ from the TPP phenyl group, which also appeared shifted with respect to pure TPP $\left(3055 \mathrm{~cm}^{-1}\right)$. The FTIR spectrum of TPP displays several additional peaks between 600 to $1200 \mathrm{~cm}^{-1}$. The peak at $1181 \mathrm{~cm}^{-1}$ is attributed to the stretching vibration of $\mathrm{C}-\mathrm{O}$ and the peaks at 854 and $681 \mathrm{~cm}^{-1}$ were assigned to the bending vibration of C$\mathrm{H}$ of the phenyl group. However, those peaks merged into a broad band centered at $870 \mathrm{~cm}^{-1}$ in the FTIR spectra of as-produced CrP NPs. The absence of all these features in the FTIR spectra of the final NPs after annealing under $\mathrm{H}_{2} /$ Ar treatment confirmed the elimination of organic ligands from the NP surface. 


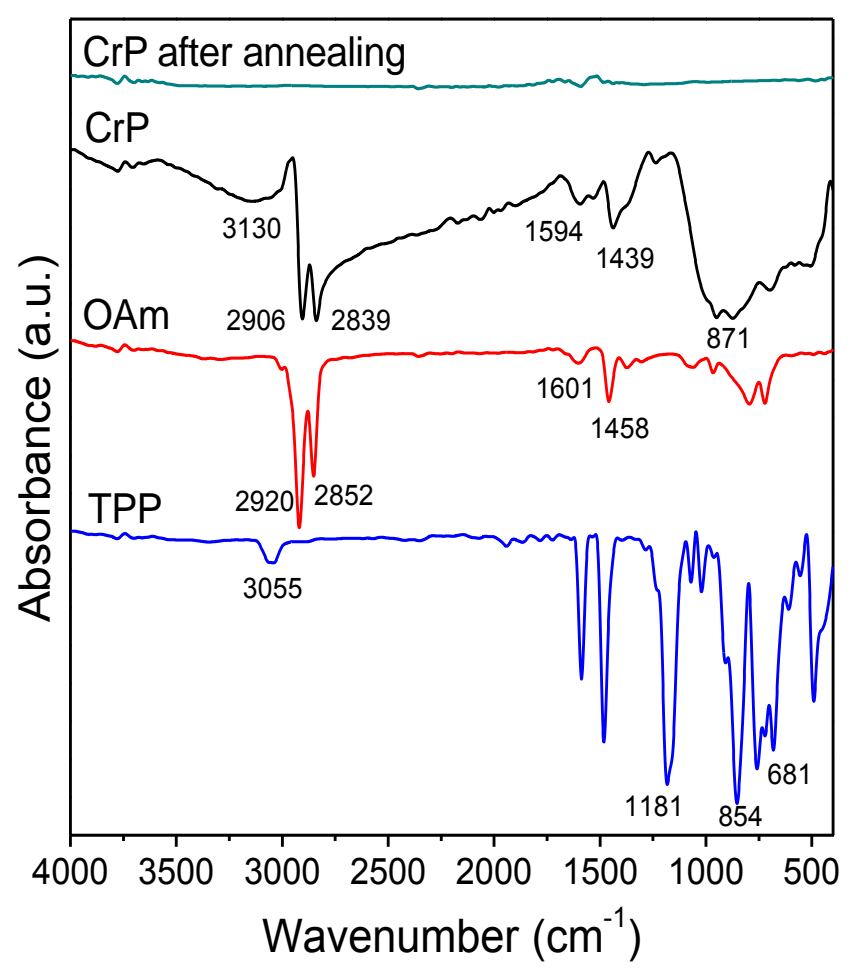

Figure 3. FTIR of OAm, TPP, as-produced CrP NPs and $\mathrm{CrP}$ after annealing at $450^{\circ} \mathrm{C}$ for $2 \mathrm{~h}$ under $\mathrm{H}_{2} / \mathrm{Ar}$.

The electrocatalytic performance toward ORR of CrP NPs was evaluated in alkaline condition. To prepare CrP catalyst, CrP NPs were supported on carbon black to a weight ratio of 3:7 (CrP NPs/C) through sonication and subsequent annealing at $450{ }^{\circ} \mathrm{C}$ under $\mathrm{H}_{2} / \mathrm{Ar}$, and then measured using a three-electrode system in $\mathrm{O}_{2}$-saturated $0.1 \mathrm{M} \mathrm{KOH}$ (see experimental section for details). CVs were initially measured both in $\mathrm{Ar}$ and in $\mathrm{O}_{2}$ saturated electrolyte, as shown in Figure 4a. Beyond the non-Faradic current characteristic of a double-layer chargedischarge, no noticeable features were observed for CrP catalyst within an Ar-saturated electrolyte. On the other hand, when the electrolyte was saturated with $\mathrm{O}_{2}$ a cathodic peak centered at $0.63 \mathrm{~V}$ was clearly detected, indicating remarkable electrocatalytic activity of CrP NPs toward ORR. Figure $\mathbf{4 b}$ shows polarization curves measured at different electrode rotation speed, from 400 to $2500 \mathrm{rpm}$. As expected, the limiting current density increased with the rotation speeds due to the promoted diffusion of oxygen at the surface of electrodes. The limiting current density of CrP NPs electrode at $0.2 \mathrm{~V}$ under $1600 \mathrm{rpm}$ reached $4.94 \mathrm{~mA} \mathrm{~cm}{ }^{-2}$, with a half-potential of $0.65 \mathrm{~V}$ and an onset potential of $0.8 \mathrm{~V}$. These values were comparable to those obtained with a commercial Pt/C electocatalyst, which provided a limiting current density of $5.63 \mathrm{~mA} \mathrm{~cm}-2$ with a half-potential of $0.79 \mathrm{~V}$ and an onset potential of $0.95 \mathrm{~V}$ (Figure $4 \mathrm{~d}$ ).

Meanwhile, the excellent ORR activity of the CrP electrocatalysts was further revealed through small Tafel slopes, 
$64 \mathrm{mV} \mathrm{dec}^{-1}$, indicating a high sensitive of the electric current response to the applied potential and a favorable kinetics for CrP catalyst (inset in figure 4d). Notice that CrP NPs were supported on low-cost commercial carbon in the present work, thus the measured catalytic performance did not rely on the electrocatalytic activity of doped graphenes [45-47], carbon nanotubes [48-51] or highly ordered porous carbon matrices [52,53] (table S1).

The ORR kinetics of the CrP electrode was further analyzed by the Koutecky-Levich plots calculated from the measured LSV curves. As shown in Figure 4c, the Koutecky-Levich plots exhibited good linearity and approximately parallel dependences of the inverse of the current density with the square root of the rotation speed for applied voltages in the range from 0.2 to $0.6 \mathrm{~V}$. This indicated first-order reaction kinetics with the oxygen concentration and denoted that, within the studied range, electron transfer numbers for ORR did not depend on the applied potential [54,55]. The calculated electron transfer number for CrP electrocatalysts toward ORR was from 3.8 to 4.1 , showing a high selectivity toward total oxygen reduction and the dominance of the one-step, 4-electron oxygen reduction pathway, in the ORR.

Stability was assessed by chronoamperometric analysis (Figure 4e). CrP/C-based electrodes exhibited much higher stabilities than Pt/C toward ORR in $0.1 \mathrm{M} \mathrm{KOH}$, with a negligible degradation even after $60 \mathrm{~h}$ of continuous operation. In contrast, during the same operation time, $\mathrm{Pt} / \mathrm{C}$ electrodes lost approximately $60 \%$ of the initial current density. Methanol tolerance measurements were performed by adding methanol to the electrolyte during the choronoamperometric measurements. As shown in Figure $\mathbf{4 f}$, the current density measured from $\mathrm{CrP} / \mathrm{C}$ electrocatalysts showed very minor changes upon the addition of methanol, up to a $3 \mathrm{M}$ concentration, into the electrolyte. The slightly changes may be actually associated to the dilution of the KOH electrolyte when methanol was added to the solution. This was confirmed by a similar current density change upon addition of the same amount of $\mathrm{H}_{2} \mathrm{O}$ into the solution, proving a virtually no influence of methanol in the electrocatalytic ORR on $\mathrm{CrP}$ baed electrodes. Overall, CrP-baed electrodes showed much higher stabilities than commercial Pt/ electrocatalysts and other electrocatalysts previously reported (Table S1). 

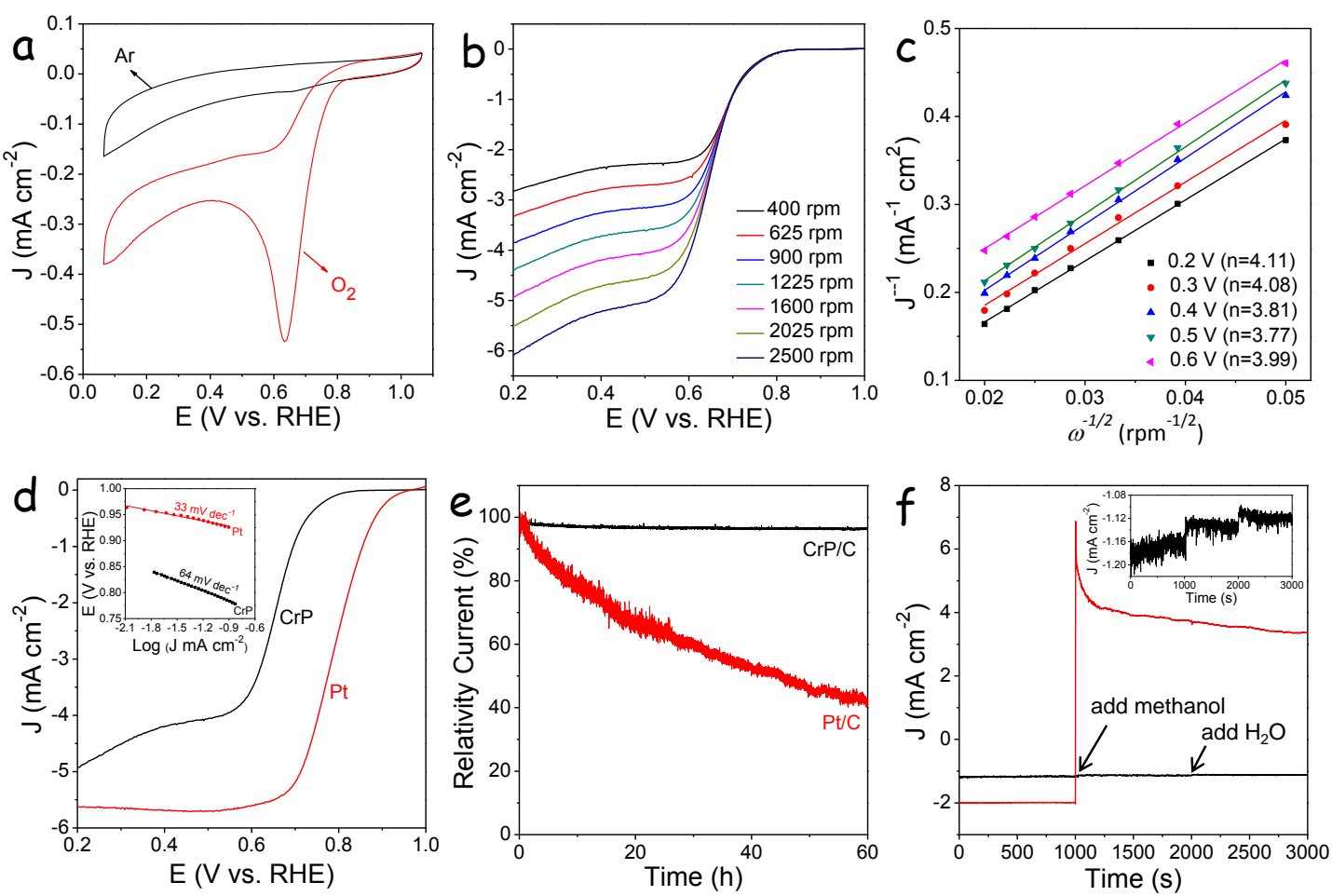

Figure 4. (a) $\mathrm{CV}$ curves of $\mathrm{CrP} / \mathrm{C}$ in $\mathrm{Ar}$-and $\mathrm{O}_{2}$-saturated $0.1 \mathrm{M} \mathrm{KOH}$ solution. (b) LSV curves of $\mathrm{CrP} / \mathrm{C}$ at various rotating rates in $\mathrm{O}_{2}$-saturated $0.1 \mathrm{M} \mathrm{KOH}$ solution at a scan rates of $5 \mathrm{mV} \mathrm{s}^{-1}$. (c) The corresponding K-L plots. (d) The comparison of LSV for CrP/C and commercial $20 \% \mathrm{Pt} / \mathrm{C}$, insertion is the Tafel plots of $\mathrm{CrP} / \mathrm{C}$ and $\mathrm{Pt} / \mathrm{C}$ catalysts. (e) chronoamperometric measurements of $\mathrm{CrP} / \mathrm{C}$ and commercial $\mathrm{Pt} / \mathrm{C}$ in $0.1 \mathrm{M} \mathrm{KOH}$ solution at $0.57 \mathrm{~V}$ versus $\mathrm{RHE}$ in $\mathrm{O}_{2}$-saturated $0.1 \mathrm{M} \mathrm{KOH}$ solution with a rotating rates of $200 \mathrm{rpm}$. (f) Methanol tolerance evaluation of $\mathrm{CrP} / \mathrm{C}$ and commercial $\mathrm{Pt} / \mathrm{C}$ in $\mathrm{O}_{2}$-saturated $0.1 \mathrm{M} \mathrm{KOH}$ solution, for comparison, same amount of $\mathrm{H}_{2} \mathrm{O}$ was added at 2000 s. Insertion showed enlarged data for $\mathrm{CrP} / \mathrm{C}$ catalyst.

First-principles calculations (DFT) were carried out to elucidate the process and parameters behind the exceptional ORR catalytic performance of $\mathrm{CrP}$ and to determine the rate limiting step. In alkaline solution, the kinetically most favorable reaction pathway for the ORR process on $\mathrm{CrP}$ was considered to be the $\mathrm{O}+\mathrm{OH}$ dissociation pathway, which can be divided into four steps [55]: i) adsorption of an $\mathrm{O}_{2}$ molecule onto $\mathrm{CrP}$ surface site $\left(\mathrm{O}_{2}{ }^{*}\right)$; ii) reduction of $\mathrm{O}_{2}{ }^{*}$ with $\mathrm{H}_{2} \mathrm{O}^{*}$ to an $\mathrm{OOH}$ group adsorbed on the $\mathrm{CrP}$ site $\left(\mathrm{OOH}^{*}\right)$; iii) transition from $\mathrm{OOH}^{*}$ to an adsorbed $\mathrm{O} *$ atom; and iv) transition from $\mathrm{O}^{*}$ to an adsorbed $\mathrm{OH}^{*}$ (Figure 5). The optimized adsorption structures of $\mathrm{O}_{2}{ }^{*}, \mathrm{OOH}^{*}$, $\mathrm{OH}^{*}$ and $\mathrm{O}^{*}$ on $\mathrm{CrP}$ catalyst and their adsorption energies were shown in Figure S2. The adsorption of $\mathrm{O}_{2}$ on $\mathrm{CrP}$, the first step in the ORR, was the first investigated. $\mathrm{O}_{2}$ strongly chemisorbs on $\mathrm{CrP}$. The $\mathrm{O}_{2}$ end-on adsorption was the most stable configuration, with very low adsorption energy, $-3.01 \mathrm{eV}$. The notable strength of the adsorption was corroborated by the large elongation of the $\mathrm{O}=\mathrm{O}$ bond length $(\mathrm{dO}=\mathrm{O}=1.50 \AA$ ) relative to the gas-phase value 
( $\mathrm{dO}=\mathrm{O}=1.23 \AA$ ), making it easier for the $\mathrm{O}=\mathrm{O}$ bond to be broken. These highly activated $\mathrm{O}_{2}$ * already pointed toward a potentially high ORR catalytic activity. Furthermore, since $\mathrm{H}_{2} \mathrm{O}_{2}$ is directly dissociated to two $\mathrm{OH}$ radicals, less efficient 2e- pathway will not occur during the ORR process. Figure 5 shows the free energy diagrams for $4 \mathrm{e}-$ oxygen reduction process of $\mathrm{CrP}$ in alkaline medium. Two pathways were considered: i) $\mathrm{O}_{2}$ dissociation (Figure 5a); and ii) $\mathrm{O}_{2}$ hydrogenation (Figure 5b). As shown in Figure 5, O-O breaking processes $\left(\mathrm{O}_{2} \rightarrow 2 \mathrm{O}^{*}\right.$ or $\mathrm{O}_{2} *+\mathrm{H}_{2} \mathrm{O}^{*} \rightarrow$ $\mathrm{OOH}^{*}+\mathrm{OH}^{*}$ ) are down-hill. On the other hand, $\mathrm{OH}^{*} \rightarrow \mathrm{OH}$ - is the main uphill process owing to the strong adsorption of $\mathrm{OH}$ to $\mathrm{CrP}(\sim 4 \mathrm{eV})$, which suggests that this is the ORR rate-limiting step.
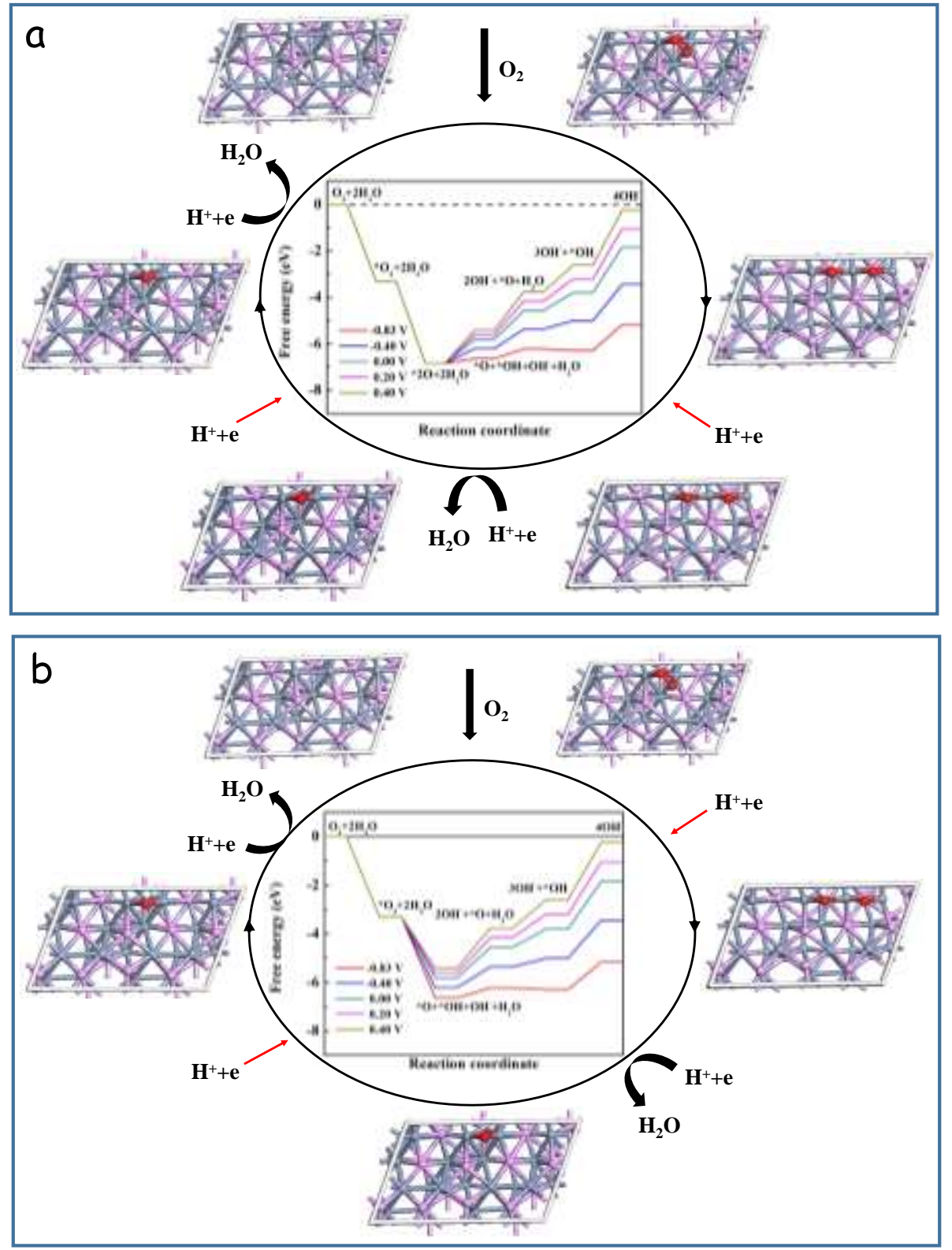

Figure 5. Free-energy diagrams for the reduction of $\mathrm{O}_{2}$ at different electrode potential in alkaline medium on $\mathrm{CrP}$ for (a) $\mathrm{O}_{2}$ dissociation pathway and (b) $\mathrm{O}_{2}$ hydrogenation pathway. 


\section{CONCLUSION}

In summary, we successfully prepared monodispersed CrP NCs via a facile one-step colloidal synthetic strategy. Compared with presented CVD method, our method could allow producing CrP with nanometric particle size and with a very high throughput and material yield. CrP NPs were mixed with carbon to prepare electrocatalysts for the ORR. CrP-based electrocatalysts demonstrated a high activity and stability toward ORR in an alkaline electrolyte and a high tolerance to methanol. DFT calculation revealed a strong adsorption of $\mathrm{O}_{2}$ on the surface of CrP NPs which facilitated cleavage. On the other hand, the $\mathrm{OH}^{-}$desorption was considered the rate limiting step in the ORR reaction. Overall, $\mathrm{CrP}$ was demonstrated as a new low-cost and earth abundant ORR electrocatalyst with high technological potential in alkaline fuel cells and metal air batteries.

\section{Acknowledgements}

This work was supported by the European Regional Development Funds and the Spanish MINECO project SEHTOP (ENE2016-77798-C4- 3-R). T. Zhang and J. Arbiol acknowledge funding from Generalitat de Catalunya 2017 SGR 327 and the Spanish MINECO project ANAPHASE (ENE2017-85087-C3). ICN2 acknowledges support from the Severo Ochoa Programme (SEV-2013-0295) and is funded by the CERCA Programme / Generalitat de Catalunya. J. Llorca is a Serra Hunter Fellow and is grateful to ICREA Academia program and grants MINECO/FEDER ENE201563969-R and GC 2017 SGR 128. J. Liu, X. Yu and R. Du thank the China Scholarship Council for scholarship support. M. Meyns acknowledges a Juan de la Cierva formación grant by the Spanish MINECO.

\section{REFERENCES}

1. Hernandez-Fernandez, Patricia, Federico Masini, David N. McCarthy, Christian E. Strebel, Daniel Friebel, Davide Deiana, Paolo Malacrida et al. "Mass-selected nanoparticles of Pt $x$ Y as model catalysts for oxygen electroreduction." Nature chemistry 6, no. 8 (2014): 732.

2. Wang, Yan-Jie, Nana Zhao, Baizeng Fang, Hui Li, Xiaotao T. Bi, and Haijiang Wang. "Carbon-supported Pt-based alloy electrocatalysts for the oxygen reduction reaction in polymer electrolyte membrane fuel cells: particle size, shape, and composition manipulation and their impact to activity."Chemical reviews 115, no. 9 (2015): 3433-3467.

3. Huang, Xiaoqing, Zipeng Zhao, Liang Cao, Yu Chen, Enbo Zhu, Zhaoyang Lin, Mufan Li et al. "High-performance transition metal-doped Pt3Ni octahedra for oxygen reduction reaction." Science 348, no. 6240 (2015): 1230-1234. 
4. Meng, Chao, Tao Ling, Tian- Yi Ma, Hui Wang, Zhenpeng Hu, Yue Zhou, Jing Mao, Xi- Wen Du, Mietek Jaroniec, and Shi- Zhang Qiao. "Atomically and electronically coupled Pt and CoO hybrid nanocatalysts for enhanced electrocatalytic performance." Advanced Materials 29, no. 9 (2017): 1604607.

5. Tong, Yun, Pengzuo Chen, Tianpei Zhou, Kun Xu, Wangsheng Chu, Changzheng Wu, and Yi Xie. "A Bifunctional Hybrid Electrocatalyst for Oxygen Reduction and Evolution: Cobalt Oxide Nanoparticles Strongly Coupled to B, NDecorated Graphene." Angewandte Chemie 129, no. 25 (2017): 7227-7231.

6. Zhou, Ye, Shibo Xi, Jingxian Wang, Shengnan Sun, Chao Wei, Zhenxing Feng, Yonghua Du, and Zhichuan J. Xu. "Revealing the Dominant Chemistry for Oxygen Reduction Reaction on Small Oxide Nanoparticles." ACS Catalysis 8, no. 1 (2017): 673-677.

7. Yang, Wenxiu, Xiangjian Liu, Xiaoyu Yue, Jianbo Jia, and Shaojun Guo. "Bamboo-like carbon nanotube/Fe3C nanoparticle hybrids and their highly efficient catalysis for oxygen reduction." Journal of the American Chemical Society137, no. 4 (2015): 1436-1439.

8. Wang, Hao, Cheng Sun, Yingjie Cao, Juntong Zhu, Yuan Chen, Jun Guo, Jie Zhao, Yinghui Sun, and Guifu Zou. "Molybdenum carbide nanoparticles embedded in nitrogen-doped porous carbon nanofibers as a dual catalyst for hydrogen evolution and oxygen reduction reactions." Carbon114 (2017): 628-634.

9. Meganathan, Mayilvel Dinesh, Shun Mao, Taizhong Huang, and Guoxin Sun. "Reduced graphene oxide intercalated Co $2 \mathrm{C}$ or Co $4 \mathrm{~N}$ nanoparticles as an efficient and durable fuel cell catalyst for oxygen reduction." Journal of Materials Chemistry A 5, no. 6 (2017): 2972-2980.

10. Hu, Kui, Zhaohui Xiao, Yi Cheng, Dafeng Yan, Ru Chen, Jia Huo, and Shuangyin Wang. "Iron phosphide/N, Pdoped carbon nanosheets as highly efficient electrocatalysts for oxygen reduction reaction over the whole $\mathrm{pH}$ range."Electrochimica Acta 254 (2017): 280-286.

11. Yang, Shuang, Qun He, Changda Wang, Hongliang Jiang, Chuanqiang Wu, Youkui Zhang, Teng Zhou, Yu Zhou, and Li Song. "Confined bimetallic phosphide within P, N co-doped carbon layers towards boosted bifunctional oxygen catalysis."Journal of Materials Chemistry A 6, no. 24 (2018): 11281-11287.

12. Wang, Qin, Yao Fan, Keke Wang, Haimin Shen, Guijie Li, Haiyan Fu, and Yuanbin She. "Hierarchical tubular structures composed of CoPx and carbon nanotubes: Highly effective electrocatalyst for oxygen reduction." Carbon 130 (2018): 241-249. 
13. Razmjooei, Fatemeh, Chanho Pak, and Jong- Sung Yu. "Phase Diversity of Nickel Phosphides in Oxygen Reduction Catalysis." ChemElectroChem 5, no. 14 (2018): 1985-1994.

14. Kucernak, Anthony RJ, K. F. Fahy, and VN Naranammalpuram Sundaram. "Facile synthesis of palladium phosphide electrocatalysts and their activity for the hydrogen oxidation, hydrogen evolutions, oxygen reduction and formic acid oxidation reactions." Catalysis Today 262 (2016): 48-56.

15. Doan-Nguyen, Vicky VT, Sen Zhang, Edward B. Trigg, Rahul Agarwal, Jing Li, Dong Su, Karen I. Winey, and Christopher B. Murray. "Synthesis and X-ray characterization of cobalt phosphide (Co2P) nanorods for the oxygen reduction reaction." ACS nano 9, no. 8 (2015): 8108-8115.

16. Yang, Hongchao, Yejun Zhang, Feng Hu, and Qiangbin Wang. "Urchin-like CoP nanocrystals as hydrogen evolution reaction and oxygen reduction reaction dual-electrocatalyst with superior stability." Nano letters 15, no. 11 (2015): 7616-7620.

17. Teller, Hanan, Olga Krichevski, Meital Gur, Aharon Gedanken, and Alex Schechter. "Ruthenium phosphide synthesis and electroactivity toward oxygen reduction in acid solutions." ACS Catalysis 5, no. 7 (2015): $4260-4267$.

18. Falkowski, Joseph, Concanon, Nolan, Yan, Bing, Surendranath, Yogesh, " Heazlewoodite, $\mathrm{Ni}_{3} \mathrm{~S}_{2}$ : A Potent Catalyst for Oxygen Reduction to Water under Benign Conditions ." J. Am. Chem. Soc. 137, no. 25 (2015): 7978-7981.

19. Zhang, Jintao, Liangti Qu, Gaoquan Shi, Jiangyong Liu, Jianfeng Chen, and Liming Dai. "N, P- Codoped Carbon Networks as Efficient Metal- free Bifunctional Catalysts for Oxygen Reduction and Hydrogen Evolution Reactions."Angewandte Chemie International Edition 55, no. 6 (2016): 2230-2234.

20. Liu, Xien, and Liming Dai. "Carbon-based metal-free catalysts." Nature Reviews Materials 1, no. 11 (2016): 16064

21. Dai, Liming, Yuhua Xue, Liangti Qu, Hyun-Jung Choi, and Jong-Beom Baek. "Metal-free catalysts for oxygen reduction reaction." Chemical reviews 115, no. 11 (2015): 4823-4892.

22. Blackman, Christopher S., Claire J. Carmalt, Troy D. Manning, Ivan P. Parkin, Leonardo Apostolico, and Kieran C. Molloy. "Low temperature deposition of crystalline chromium phosphide films using dual-source atmospheric pressure chemical vapour deposition." Applied surface science 233, no. 1-4 (2004): 24-28.

23. Motojima, Seiji, and Tokihisa Higashi. "Deposition and hallow crystal growth of CrP and Cr3P by CVD 
process." Journal of crystal growth 71, no. 3 (1985): 639-647.

24. Myers, C. E., G. A. Kisacky, and J. K. Klingert. "Vaporization Behavior of Chromium Phosphides The Solid TwoPhase Regions, and." Journal of The Electrochemical Society 132, no. 1 (1985): 236-238.

25. Lone, Ikram Un Nabi, and M. Mohamed Sheik Sirajuddeen. "Half metallic ferromagnetism in gallium and zinc doped chromium phosphide: First principles calculations." Materials Chemistry and Physics 203 (2018): 65-72.

26. Nagai, T., M. Miyake, H. Kimura, and M. Maeda. "Determination of Gibbs free energy of formation of Cr3P by double Knudsen cell mass spectrometry." The Journal of Chemical Thermodynamics 40, no. 3 (2008): 471-475.

27. Zaitsev, A. I., N. E. Shelkova, A. D. Litvina, B. M. Mogutnov, and Zh V. Dobrokhotova. "Thermodynamic properties and phase equilibria in the Cr-P system." Journal of phase equilibria 19, no. 3 (1998): 191-199.

28. Amutha, B., and R. Velavan. "Half-metallicity in Rhodium doped Chromium Phosphide: An ab-initio study." $2018,7,4$

29. Liu, Junfeng, Michaela Meyns, Ting Zhang, Jordi Arbiol, Andreu Cabot, and Alexey Shavel. "Triphenyl Phosphite as the Phosphorus Source for the Scalable and Cost-Effective Production of Transition Metal Phosphides." Chemistry of Materials 30, no. 5 (2018): 1799-1807.

30. Liu, Junfeng, Zhenxing Wang, Jeremy David, Jordi Llorca, Junshan Li, Xiaoting Yu, Alexey Shavel, Jordi Arbiol, Michaela Meyns, and Andreu Cabot. "Colloidal Ni 2- x Co x P nanocrystals for the hydrogen evolution reaction." Journal of Materials Chemistry A (2018), 11453-11462.

31. G. Kresse and J. Furthmuller. "Efficiency of ab-initio total energy calculations for metals and semiconductors using a plane-wave basis set." Comp. Mater. Sci., 1996, 6, 15-50.

32. G. Kresse and J. Hafner. "Ab initio molecular dynamics for liquid metals." Phys. Rev. B, 1993, 47, 558-561.

33. G. Kresse and J. Hafner. "Ab initio molecular-dynamics simulation of the liquid-metal-amorphoussemiconductor transition in germanium." Phys. Rev. B, 1994, 49, 14251-14269.

34. G. Kresse and J. Furthmuller. "Efficient iterative schemes for ab initio total-energy calculations using a planewave basis set." Phys. Rev. B, 1996, 54, 11169-11186.

35. P. E. Blochle. "Projector augmented-wave method." Phys. Rev. B, 1994, 50, 17953-17979. 
36. G. Kresse, D. Joubert. "From ultrasoft pseudopotentials to the projector augmented-wave method." Phys. Rev. $B, 1999, \mathbf{5 9}, 1758-1775$.

37. J. P. Perdew, K. Burke and M. Ernzerhof. “Generalized gradient approximation made simple." Phys. Rev. Lett., 1996, 77, 3865-3868.

38. H. J. Monkhorst and J. D. Pack. "Special points for Brillouin-zone integrations." Phys. Rev. B, 1976, 13, 51885192.

39. Nørskov, J. K.; Rossmeisl, J.; Logadottir, A.; Lindqvist, L.; Kitchin, J. R.; Bligaard, T.; Jónsson, H., Origin of the Overpotential for Oxygen Reduction at a Fuel-Cell Cathode. J. Phys. Chem. B 2004, 108, 17886-17892.

40. Yu, L.; Pan, X.; Cao, X.; Hu, P.; Bao, X., Oxygen Reduction Reaction Mechanism on Nitrogen-Doped Graphene: A Density Functional Theory Study. J. Catal. 2011, 282, 183-190.

41. Http://Webbook.Nist.Gov/Chemistry/. see Supplemental Material at http://webbook.nist.gov/chemistry/.

42. Asteman, H., J-E. Svensson, M. Norell, and L-G. Johansson. "Influence of water vapor and flow rate on the hightemperature oxidation of 304L; effect of chromium oxide hydroxide evaporation." Oxidation of Metals 54, no. 1-2 (2000): 11-26.

43. Pettersson, R. F. A., J. Enecker, and L. Liu. "Role of nickel in the oxidation of Fe-Cr-Ni alloys in air-water vapour atmospheres." Materials at High Temperatures 22, no. 3-4 (2005): 269-281.

44. Bao, Binbin, Jinglei Liu, Hong Xu, Bo Liu, Kai Zhang, and Zhaorong Jin. "Insight into a high temperature selective oxidation of HP40 alloy under a H 2-H 2 O environment." RSC Advances 7, no. 14 (2017): 8589-8597.

45. Pendashteh, Afshin, Jesus Palma, Marc Anderson, and Rebeca Marcilla. "NiCoMnO4 nanoparticles on N-doped graphene: Highly efficient bifunctional electrocatalyst for oxygen reduction/evolution reactions." Applied Catalysis B: Environmental 201 (2017): 241-252.

46. Wang, Qichen, Yongpeng Lei, Zhiyan Chen, Nan Wu, Yaobing Wang, Bing Wang, and Yingde Wang. "Fe/Fe 3 C@ C nanoparticles encapsulated in N-doped graphene-CNTs framework as an efficient bifunctional oxygen electrocatalyst for robust rechargeable Zn-air batteries." Journal of Materials Chemistry A 6, no. 2 (2018): 516526.

47. Wu, Zhong-Shuai, Shubin Yang, Yi Sun, Khaled Parvez, Xinliang Feng, and Klaus Müllen. "3D nitrogen-doped 
graphene aerogel-supported Fe304 nanoparticles as efficient electrocatalysts for the oxygen reduction reaction." Journal of the American Chemical Society 134, no. 22 (2012): 9082-9085.

48. Wei, Changting, Mengxia Shen, Kelong Ai, and Lehui Lu. "Transformation from FeS/Fe3C nanoparticles encased S, N dual doped carbon nanotubes to nanosheets for enhanced oxygen reduction performance." Carbon 123 (2017): 135-144.

49. Zeng, Shanshan, Fucong Lyu, Hongjiao Nie, Yawen Zhan, Haidong Bian, Yayuan Tian, Zhe Li, Aiwu Wang, Jian Lu, and Yang Yang Li. "Facile fabrication of N/S-doped carbon nanotubes with Fe 304 nanocrystals enchased for lasting synergy as efficient oxygen reduction catalysts." Journal of Materials Chemistry A 5, no. 25 (2017): 1318913195.

50. Han, Xiaopeng, Xiaoyu Wu, Cheng Zhong, Yida Deng, Naiqin Zhao, and Wenbin Hu. "NiCo2S4 nanocrystals anchored on nitrogen-doped carbon nanotubes as a highly efficient bifunctional electrocatalyst for rechargeable zinc-air batteries."Nano Energy 31 (2017): 541-550.

51. Zhao, Shuai, Brian Rasimick, William Mustain, and Hui Xu. "Highly durable and active Co 3 O nanocrystals supported on carbon nanotubes as bifunctional electrocatalysts in alkaline media." Applied Catalysis B: Environmental 203 (2017): 138-145.

52. Zhu, Jingyue, Dan Xu, Cancan Wang, Wenjing Qian, Jun Guo, and Feng Yan. "Ferric citrate-derived N-doped hierarchical porous carbons for oxygen reduction reaction and electrochemical supercapacitors." Carbon 115 (2017): 1-10.

53. Chen, Yuanjun, Shufang Ji, Yanggang Wang, Juncai Dong, Wenxing Chen, Zhi Li, Rongan Shen et al. "Isolated single iron atoms anchored on $\mathrm{N}$ - doped porous carbon as an efficient electrocatalyst for the oxygen reduction reaction." Angewandte Chemie International Edition 56, no. 24 (2017): 6937-6941.

54. Chen, Binling, Rong Li, Guiping Ma, Xinglong Gou, Yanqiu Zhu, and Yongde Xia. "Cobalt sulfide/N, S codoped porous carbon core-shell nanocomposites as superior bifunctional electrocatalysts for oxygen reduction and evolution reactions."Nanoscale 7, no. 48 (2015): 20674-20684.

55. Yu, Xuelian, Da Wang, Jingjing Liu, Zhishan Luo, Ruifeng Du, Li-Min Liu, Guangjin Zhang, Yihe Zhang, and Andreu Cabot. "Cu2ZnSnS4 Nanocrystals as Highly Active and Stable Electrocatalysts for the Oxygen Reduction Reaction." The Journal of Physical Chemistry C 120, no. 42 (2016): 24265-24270. 